\title{
Real-Time Color-Tunable Electroluminescence From Stacked Organic LEDs Using Independently Addressable Middle Electrode
}

\author{
H. M. Zhang and Wallace C. H. Choy, Senior Member, IEEE
}

\begin{abstract}
Independently controllable stacked organic light emitting devices (OLEDs) are fabricated by using interconnecting electrode of $\mathrm{Al}(2 \mathrm{~nm}) / \mathrm{WO}_{3}(3 \mathrm{~nm}) / \mathrm{Au}(16 \mathrm{~nm})$ for connecting two primary color OLED units of blue and red. The middle electrode simultaneously functions as the cathode and anode for the bottom and top units respectively with a feature of over $60 \%$ optical transmission in a wide wavelength range from 500 to $700 \mathrm{~nm}$ such that the color can be tuned in real time from red to blue by changing the bias voltage to the two units. The undistorted primary colors and high efficiency have been obtained through optimizing structure and properly arranging the ordering of the blue and red subpixels.
\end{abstract}

Index Terms-High optical transmission electrode, intermediate electrode, real-time color tuning, stacked organic light-emitting devices (SOLEDs).

\section{INTRODUCTION}

$\mathbf{O}$ RGANIC light-emitting devices (OLEDs) have gained interest for their promising applications in full-color flat panel display [1]-[3]. For making such full-color displays, schemes such as multiple-emitting layers [4], microcavity structure [5], [6], voltage-controlled dye doping [7], and inserting carrier blocking layers [8] for achieving color tunability have been proposed. Among the methods, vertically stacked red, green, and blue-emitting OLEDs have led to a threefold increase in resolution and display fill factor as compared to the other traditional side-by-side subpixel arrangement [4]. The emission from a subpixel transmits through the vertically adjacent units in the independently addressable stacked OLEDs (SOLEDs), thus the high transparency of the interconnecting electrodes with appropriate carrier injection properties are necessary. Some attempts have been made on exploring high-performance interconnecting electrodes. For the independently controllable SOLEDs, the interconnecting layer should simultaneously function as an anode for one unit and as a cathode for another unit besides the necessity of good transmission. To realize

\footnotetext{
Manuscript received January 13, 2008; revised March 27, 2008. This work was supported in part by the UDF under a grant, in part by the Strategic Research Grant in Organic Optoelectronics of Hong Kong University, and in part by the Research Grant Council of the Hong Kong Special Administrative Region, China, under Grant HKU 7124/05E.

H. M. Zhang is with the Department of Electrical and Electronic Engineering, University of Hong Kong, Hong Kong, on leave from the State Key Laboratory of Polymer Physics and Chemistry, Changchun Institute of Applied Chemistry, Chinese Academy of Sciences, Changchun 130022, China.

W. C. H. Choy is with the Department of Electrical and Electronic Engineering, University of Hong Kong, Hong Kong (e-mail: chchoy@eee.hku.hk).

Digital Object Identifier 10.1109/LPT.2008.925190
}

the functions, at present a semitransparent metal layer or a transparent indium-tin oxide (ITO), such as, $\mathrm{Mg}: \mathrm{Ag}$-indium tin oxide (ITO) [9], CuPc-ITO [10], and LiF-Ca-Ag [11], are generally used. However, there may be potential thin-film damage by using sputtering of ITO. Meanwhile, the typically low transmittance of the metal electrodes may limit the improvement of the device performance. Therefore, it is desirable to develop the intermediate controllable metal electrodes with the targets of not only improving the transmission but also enhancing the efficiency of the SOLEDs; however, the studies on the high transmission metal electrodes are limited.

In this letter, we shall use controllable middle electrodes of $\mathrm{Al}(2 \mathrm{~nm}) / \mathrm{WO}_{3}(3 \mathrm{~nm}) / \mathrm{Au}(16 \mathrm{~nm})$ to realize color-tunable independently voltage-controlling SOLEDs. Besides the good transmission of the middle electrodes of above $60 \%$ over a wide wavelength range from 500 to $713 \mathrm{~nm}$ with a peak value of $62.5 \%$, the metal Au layer on top of $\mathrm{WO}_{3}$ layer serves as the hole-injection contact to the top unit and the thin metal $\mathrm{Al}$ serves as the electron injecting contact for the bottom OLED. The $\mathrm{MoO}_{3}$ treatment of the Au layer surface can provide a good hole injection. As a consequence, each emitting unit in the SOLED can be independently addressable such that the color of pixel output can be continuously tuned from red through blue in real time. The undistorted primary color and high efficiency have been obtained through optimizing the structure and properly arranging the ordering of the blue and red subpixels. The efficiency of each emitting unit is close to the measured efficiency of the corresponding single-unit red and blue OLEDs.

\section{EXPERIMENT}

Fig. 1(a) shows the schematic diagrams of SOLEDs used in this letter. In the SOLEDs, the two emissive units consist of ITO/ $\mathrm{MoO}_{3}(8 \mathrm{~nm}) / \mathrm{NPB}(100 \mathrm{~nm}) / \mathrm{DSA}-\mathrm{Ph}: \mathrm{MADN}$ $(40 \mathrm{~nm}) / \mathrm{Alq}_{3}(10 \mathrm{~nm}) / \mathrm{LiF}(1 \mathrm{~nm}) / \mathrm{Al}(2 \mathrm{~nm}) / \mathrm{WO}_{3}(3 \mathrm{~nm}) / \mathrm{Au}$ $\left(\begin{array}{lll}16 & \mathrm{~nm}\end{array}\right) / \mathrm{MoO}_{3} \quad(5 \mathrm{~nm}) / \mathrm{NPB} \quad(60 \quad \mathrm{~nm}) / \mathrm{Alq}_{3}$ : DCJTB $(30 \mathrm{~nm}) / \mathrm{Alq}_{3}(30 \mathrm{~nm}) / \mathrm{LiF}(1 \mathrm{~nm}) / \mathrm{Al}(150 \mathrm{~nm})$. Apart from sequencing red top and blue bottom units as the SOLED, the red and blue units were also exchanged to study the effect of device sequence. All devices were fabricated on indium tin oxide (ITO) coated glass with a sheet resistance of $10 \Omega$ and thermally deposited $\mathrm{LiF}-\mathrm{Al}$ was used as a cathode. ITO substrate was cleaned and treated by $\mathrm{O}_{2}$ plasma. The deposition was carried out at pressure less than $3 \times 10^{-4} \mathrm{~Pa}$ without vacuum breaks. The organics and metal oxide were evaporated at the rate in a range of $0.2-0.3 \mathrm{~nm} / \mathrm{s}$, and the metals were evaporated at the rate of $0.8-1 \mathrm{~nm} / \mathrm{s}$. The devices have 

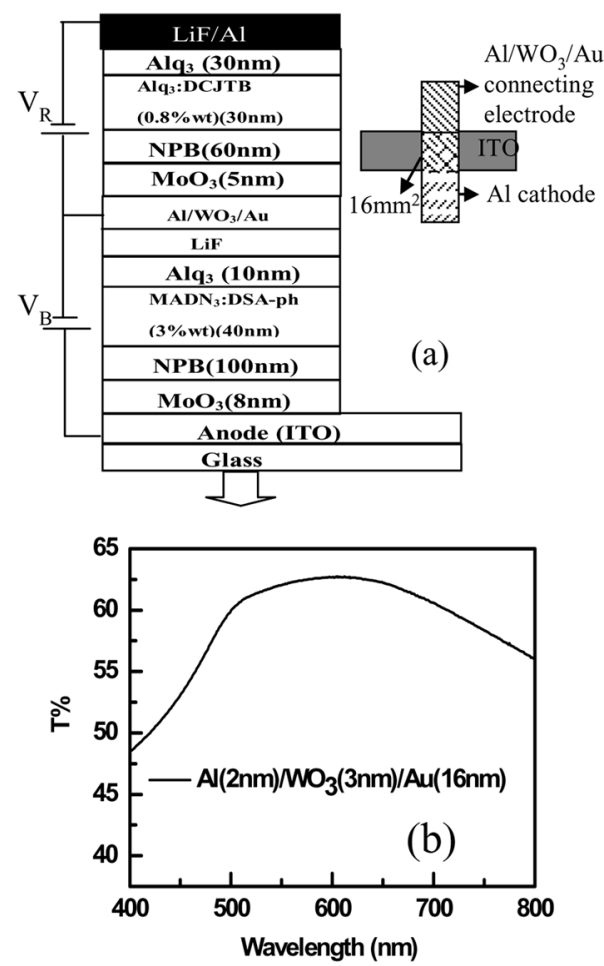

Fig. 1. (a) Schematic diagram of independently voltage controllable SOLEDs. (b) Transmittance spectrum of $\mathrm{Al}(2 \mathrm{~nm}) / \mathrm{WO} 3(3 \mathrm{~nm}) / \mathrm{Au}(16 \mathrm{~nm})$.

an emissive area of $16 \mathrm{~mm}^{2}$. The current-voltage-brightness characteristics were recorded using a computer-controlled sourcemeter (Keithley 2400) and multimeter (Keithley 2000) with a calibrated silicon photodiode. The EL spectra were measured by JY SPEX CCD3000 spectrometer. All the measurements were carried out in ambient atmosphere at room temperature.

\section{RESULTS AND DISCUSSION}

The SOLED is operated by common grounding the middle $\mathrm{Al}-\mathrm{WO}_{3}-\mathrm{Au}$ electrode as shown in Fig. 1(a). The top Al electrode and bottom ITO electrode are biased negatively and positively $\left(V_{R}\right.$ and $\left.V_{B}\right)$, respectively, with respect to the $\mathrm{Al}-\mathrm{WO}_{3}-\mathrm{Au}$ electrode. The output spectra from the SOLED at various drive voltages applied to the red and blue units are measured through the glass substrate and shown in Fig. 2(a). The top unit (red light), bottom unit (blue light), and corresponding (red and blue) single-unit control devices were biased at 11, 7,10 , and $6 \mathrm{~V}$, respectively, the peak wavelength of the red $(621 \mathrm{~nm})$ and blue $(461 \mathrm{~nm})$ units match their corresponding single-unit control OLEDs as shown in Fig. 2(b). The device emission spectrum can be varied by a linear combination of the red and blue colors through independently varying the driving voltage of each unit at the ratio of the drive voltage $\left(V_{R} / V_{B}\right)$ without the formation of exciplex [3]. Fig. 2(a) clearly shows that the emission spectrum from each unit in the SOLED can be tuned independently. For the blue and red emission as shown in Fig. 2(b), the full-width at half-maximum (FWHM) of the red and blue emission spectra from SOLED is $65 \mathrm{~nm}$ (red) and $58 \mathrm{~nm}$ (blue) with respect to the corresponding single-unit control device of $70 \mathrm{~nm}$ (red) and $52 \mathrm{~nm}$ (blue) without any

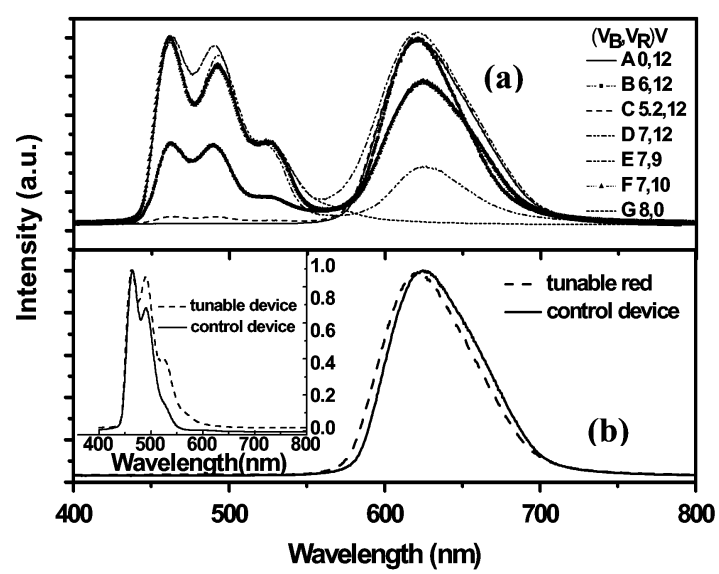

Fig. 2. (a) Emission intensity versus wavelength under various operating voltages at normal direction. (b) The emission spectra of the red light unit in the SOLEDs and the corresponding control devices biased at 11 and $10 \mathrm{~V}$, respectively. The inset is the emission spectra of the blue light unit of the SOLEDs and the corresponding control devices biased at 7 and $6 \mathrm{~V}$, respectively.

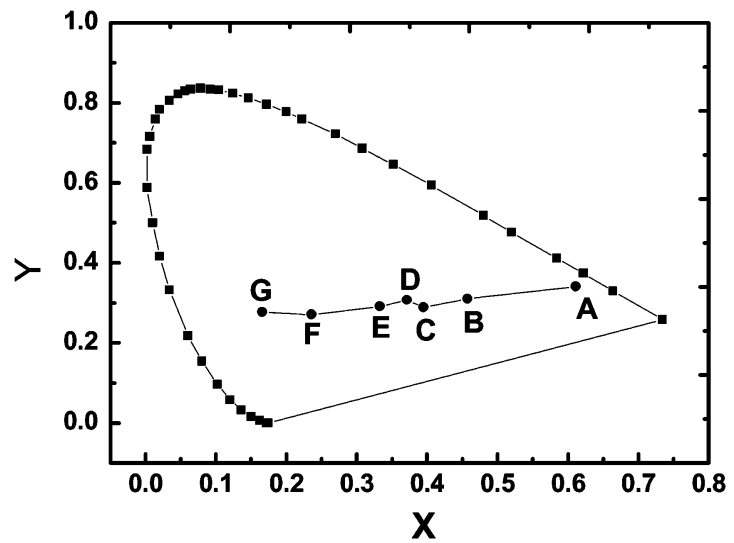

Fig. 3. The CIE color coordinates of the spectra in Fig. 2(a) with conditions A-G stated in Fig. 2(a).

significant change or obvious microcavity effect [12]. Fig. 3 shows the Commission Internationale de l'Eclairage (CIE) diagram of color tunable SOLEDs which indicates the continuous color tuning between the colors offered by the individual units in real time.

Fig. 4 shows the current efficiency versus current density curves of the red and blue emission from SOLEDs, where each unit was biased individually in the measurement. The performance of the bottom red unit and top red unit of the two sequence of SOLEDs and the control device with the same structure as the top red unit have been studied. The inset of Fig. 4 shows the blue devices. It is clearly seen that the current efficiency of each unit from SOLEDs [7.2 cd/A (red) and $3.5 \mathrm{~cd} / \mathrm{A}$ (blue)] is close to that of the corresponding single-units red ( $7 \mathrm{~cd} / \mathrm{A})$ and blue $(4 \mathrm{~cd} / \mathrm{A})$ OLED, when the red OLED is arranged as the top unit of the SOLED. In fact, the blue OLED should be arranged as the bottom unit because the blue OLED as the bottom unit has higher efficiency, as shown in the inset of Fig. 4, and the current efficiency of the red-emitting device has a minor change. Regarding the sequence of the blue and red units in SOLEDs, the transmission properties of the $\mathrm{Al}-\mathrm{WO}_{3}-\mathrm{Au}$ has to be investigated, as shown in Fig. 1(b). It is clearly shown that 


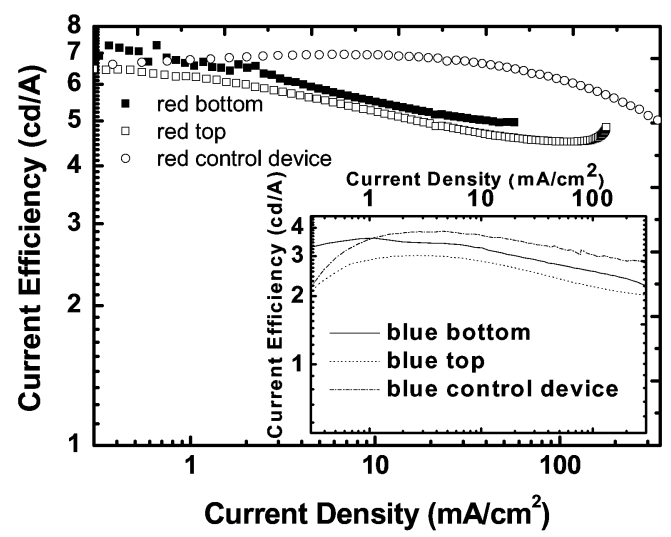

Fig. 4. The current efficiency verse current density characteristics of red light unit (top and bottom) in the two sequences of SOLEDs with the structure: $\mathrm{NPB}(60 \mathrm{~nm}) / \mathrm{Alq} 3: \operatorname{DCJTB}(30 \mathrm{~nm}) / \mathrm{Alq} 3 \quad(30 \mathrm{~nm})$ (top) and NPB(100 nm)/Alq3 : DCJTB(30 nm)/Alq3(30 nm) (bottom); The inset is that of the blue light unit (top and bottom) in the two sequences of SOLEDs with the same structure: NPB $(60 \mathrm{~nm}) / \mathrm{MADN}$ : DSA-ph(3\%) $(40 \mathrm{~nm}) / \mathrm{Alq}_{3}(10 \mathrm{~nm})$.

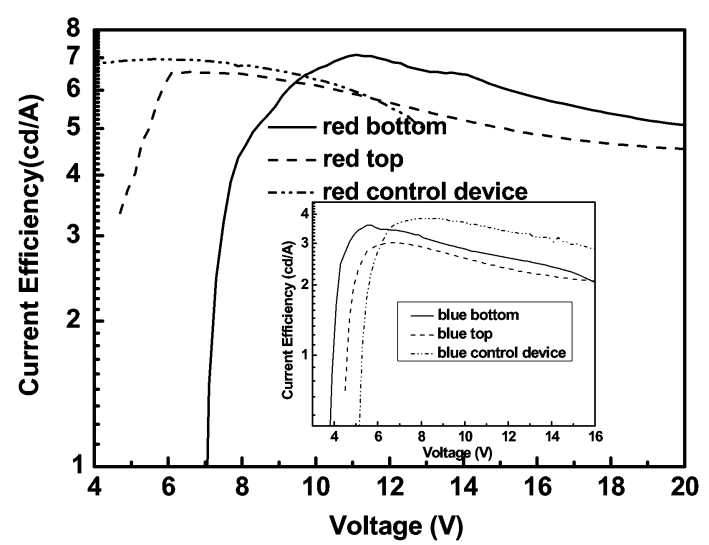

Fig. 5. The current efficiency verse applied voltage characteristics of red light unit (top and bottom) with the same structures as Fig. 4. The inset is that of the blue light unit (top and bottom) with the same structures as in Fig. 4.

the $\mathrm{Al}-\mathrm{WO}_{3}-\mathrm{Au}$ structure exhibits a good transmittance in the green-red region of 500 to $700 \mathrm{~nm}$ of over $60 \%$, favoring the red OLED as the top unit of the SOLED with lower absorption loss. The low transmittance of $\sim 52 \%$ at $450 \mathrm{~nm}$ makes the efficiency of blue OLED as the top unit of SOLED reduces (see the inset of Fig. 4), apart from the possible reabsorption of blue light in the red bottom unit in the blue-top and red-bottom SOLED. Furthermore, in many SOLEDs, the interconnecting layer is used to generate electrons and holes at an electric field. It is typically considered that the electrons are injected into bottom device and the holes are injected into top device, thus playing the role of change generation layer. Here, $\mathrm{Al}-\mathrm{WO}_{3}-\mathrm{Au}$ may be considered as a capacitor, since a layer of $\mathrm{WO}_{3}$ between $\mathrm{Al}$ and $\mathrm{Au}$ layers serves as a dielectric layer. There is no direct contact between the $\mathrm{Au}$ and $\mathrm{Al}$; thus the positive charges exist at the $\mathrm{Au}$ anode and the negative charges exist at the $\mathrm{Al}$ cathode for assisting the injection of the carriers. As a result, the efficiency is further enhanced due to the formation of more excitons in $\mathrm{Al}-\mathrm{WO}_{3}-\mathrm{Au}$ SOLEDs.
Fig. 5 and the inset figure show the current efficiency versus driving voltage curves of the red and blue emission from SOLED and the control device with the same arrangement as in Fig. 4. It can be seen that although the applied voltage is higher, the current efficiency of bottom red emission unit is slight higher than that of the upper unit and the control device. Besides, as shown in the inset of Fig. 5, the driving voltage of the blue bottom unit is lower than that of the blue top unit and the control device. As a result, putting the blue OLED as the bottom unit is reasonable for providing better performance.

\section{CONCLUSION}

In summary, an effective interconnecting structure for high efficiency SOLEDs is reported. The connecting structure is composed of a thin metal oxide sandwiched between $\mathrm{Al}$ and $\mathrm{Au}$ metals. It is clearly seen that such a connecting structure permits effective electrons and holes injection into the two adjacent emitting units. The utilization of metal oxide between $\mathrm{Al}$ and $\mathrm{Au}$ provides the interconnecting layer an over $60 \%$ transmittance in a wide wavelength range from 500 to $700 \mathrm{~nm}$, for a better transmission of red light from the blue-bottom and red-top SOLED. Furthermore, such a connecting structure can be easily fabricated by thermal evaporation for achieving real-time color-tunable SOLEDs through independently changing the biases to the two units, thus realizing high-resolution, independently addressable, stacked red-green-blue pixels for application of color displays.

\section{REFERENCES}

[1] C. W. Tang and S. A. VanSlyke, "Organic electroluminescent diodes," Appl. Phys. Lett., vol. 51, pp. 913-915, 1987.

[2] P. E. Burrows, G. Gu, V. Bulovic, Z. Shen, S. R. Forrest, and M. E. Thompson, "Achieving full-color organic light-emitting devices for lightweight, flat-panel displays," IEEE Trans. Electron Devices, vol. 44, no. 10, pp. 1188-1203, Oct. 1997.

[3] C. J. Liang and W. C. H. Choy, "Color-tunable organic light-emitting diodes by using europium organometallic complex," Appl. Phys. Lett., vol. 89, p. 251108, 2006.

[4] Z. Shen, P. E. Burrows, V. Bulovic, S. R. Forrest, and M. E. Thompson, "Three-color, tunable, organic light-emitting devices," Science, vol. 276, pp. 2009-2011, 1997.

[5] A. Dodabalapur, L. J. Rothberg, and T. M. Miller, "Color variation with electroluminescent organic semiconductors in multimode resonant cavities," Appl. Phys. Lett., vol. 65, pp. 2308-2310, 1994.

[6] W. C. H. Choy, J. H. Niu, W. L. Li, and P. C. Chui, "Voltage controlled color tunable microcavity OLEDs with enhanced color purity," J. Phys. $D$, vol. 41, p. 025106, 2008.

[7] F. Z. Wang, P. Wang, X. Fan, X. N. Dang, C. G. Zhen, D. C. Zou, E. H. Kim, D. N. Lee, and B. H. Kim, "Voltage-controlled multicolor emitting devices," Appl. Phys. Lett., vol. 89, p. 183519, 2006.

[8] W. C. H. Choy, J. H. Niu, X. W. Chen, W. L. Li, and P. C. Chui, "Effects of carrier barrier on voltage controllable color tunable OLEDs," Appl. Phys. A, vol. 89, pp. 667-671, 2007.

[9] P. E. Burrows, S. R. Forrest, S. P. Sibley, and M. E. Thompson, "Colortunable organic light-emitting devices," Appl. Phys. Lett., vol. 69, p. 2959, 1996.

[10] G. Gu, G. Parthasarathy, P. E. Burrows, P. Tian, I. G. Hill, A. Kahn, and S. R. Forrest, "Transparent stacked organic light emitting devices. I. Design principles and transparent compound electrodes," J. Appl. Phys., vol. 86, pp. 4067-4075, 1999.

[11] J. X. Sun, X. L. Zhu, H. J. Peng, M. Wong, and H. S. Kwok, "Bright and efficient white stacked organic light-emitting diodes," Organic Electron., vol. 8, pp. 305-310, 2007.

[12] W. C. H. Choy and Y. C. Ho, "Improving the viewing angle of microcavity OLEDs by using dispersive Bragg grating," Opt. Express, vol. 15 , pp. 13288-13294, 2007. 